University of New Hampshire

University of New Hampshire Scholars' Repository

Space Science Center

Institute for the Study of Earth, Oceans, and

Space (EOS)

$9-2-2005$

\title{
Gas micro-well track imaging detectors for gamma-ray astronomy
}

Peter F. Bloser

University of New Hampshire, Peter.Bloser@unh.edu

S Hunter

Goddard Space Flight Center

James M. Ryan

University of New Hampshire, James.Ryan@unh.edu

Mark L. McConnell

University of New Hampshire - Main Campus, mark.mcconnell@unh.edu

John R. Macri

University of New Hampshire - Main Campus, John.Macri@unh.edu

Follow this and additional works at: https://scholars.unh.edu/ssc

Part of the Astrophysics and Astronomy Commons

\section{Recommended Citation}

P. F. Bloser ; S. D. Hunter ; J. M. Ryan ; M. L. McConnell and J. R. Macri "Gas micro-well track imaging detectors for gamma-ray astronomy", Proc. SPIE 5898, UV, X-Ray, and Gamma-Ray Space Instrumentation for Astronomy XIV, 58980L (September 02, 2005); doi:10.1117/12.617585; http://dx.doi.org/10.1117/ 12.617585

This Conference Proceeding is brought to you for free and open access by the Institute for the Study of Earth, Oceans, and Space (EOS) at University of New Hampshire Scholars' Repository. It has been accepted for inclusion in Space Science Center by an authorized administrator of University of New Hampshire Scholars' Repository. For more information, please contact Scholarly.Communication@unh.edu. 


\title{
Gas Micro-Well Track Imaging Detectors for Gamma-ray Astronomy
}

\author{
P. F. Bloser ${ }^{a}$, S. D. Hunter ${ }^{b}$, J. M. Ryan ${ }^{a}$, M. L. McConnell ${ }^{a}$, J. R. Macri ${ }^{a}$ \\ ${ }^{a}$ Space Science Center, University of New Hampshire, Durham, NH, USA; \\ ${ }^{b}$ NASA Goddard Space Flight Center, Code 661, Greenbelt, MD, USA
}

\begin{abstract}
We describe our program to develop gas micro-well detectors (MWDs) as three-dimensional charged particle trackers for use in advanced gamma-ray telescope concepts. A micro-well detector consists of an array of individual micro-patterned gas proportional counters opposite a planar drift electrode. The well anodes and cathodes may be connected in $\mathrm{X}$ and $\mathrm{Y}$ strips, respectively, to provide two-dimensional imaging. When combined with transient digitizer electronics, which record the time signature of the charge collected in the wells of each strip, full three-dimensional reconstruction of charged-particle tracks in large gas volumes is possible. Such detectors hold great promise for advanced Compton telescope (ACT) and advanced pair telescope (APT) concepts due to the very precise measurement of charged particle momenta that is possible (Compton recoil electrons and electron-positron pairs, respectively). We present preliminary lab results, including detector fabrication, prototype electronics, and initial detector testing. We also discuss applications to the ACT and APT mission concepts, based on GEANT3 and GEANT4 simulations.
\end{abstract}

Keywords: gamma-ray astronomy, Compton telescopes, pair telescopes, gas detectors, polarimetry, Monte Carlo simulations

\section{INTRODUCTION}

The next generation of medium-energy $(0.5-50 \mathrm{MeV})$ and high-energy (30 MeV - $100 \mathrm{GeV})$ gamma-ray telescopes (Compton scatter and pair production telescopes, respectively) will require a substantial improvement in angular resolution in order to greatly improve on the sensitivity of previous and currently-planned missions. These include the medium-energy COMPTEL ${ }^{1}$ and high-energy EGRET ${ }^{2}$ telescopes on the Compton Gamma Ray Observatory, and the high-energy AGILE $^{3}$ and GLAST ${ }^{4}$ missions due to launch in the next few years. In both the medium- and high-energy cases, accurate imaging, which decreases the relative influence of background, relies on a good knowledge of the momenta of secondary particles produced in the primary gamma-ray interaction. These secondary particles are the scattered gamma-ray and recoil electron in the case of Compton scattering, and the electron-positron pairs in the case of pair production. Precisely recording these momenta also enables various background-rejection techniques and greatly increases the sensitivity of the telescope to the polarization of the incident radiation.

The angular resolution of the previous and current gamma-ray telescopes mentioned above is limited by multiple Coulomb scattering of the charged secondary particles within the detector materials which masks the particles' initial momenta. These factors have contributed to an enlarged point spread function (PSF) in current gamma-ray instruments and, in the case of pair production telescopes, have totally suppressed the polarization sensitivity. ${ }^{5}$ Improving this picture will require a low-density tracking medium with high spatial readout resolution. We therefore are investigating basing future gamma-ray instruments on micro-pattern gas detectors. ${ }^{6-9}$ Here we outline possible designs for advanced Compton and pair telescopes using gas micro-well detectors currently under development at NASA/Goddard Space Flight Center (GSFC).

Further author information: (Send correspondence to P. F. Bloser)

P. F. Bloser: E-mail: Peter.Bloser@unh.edu

UV, X-Ray, and Gamma-Ray Space Instrumentation for Astronomy XIV edited by Oswald H. W. Siegmund, Proc. of SPIE Vol. 5898 58980L, $(2005) \cdot 0277-786 \times / 05 / \$ 15 \cdot$ doi: $10.1117 / 12.617585$

Proc. of SPIE Vol. 5898 58980L-1 


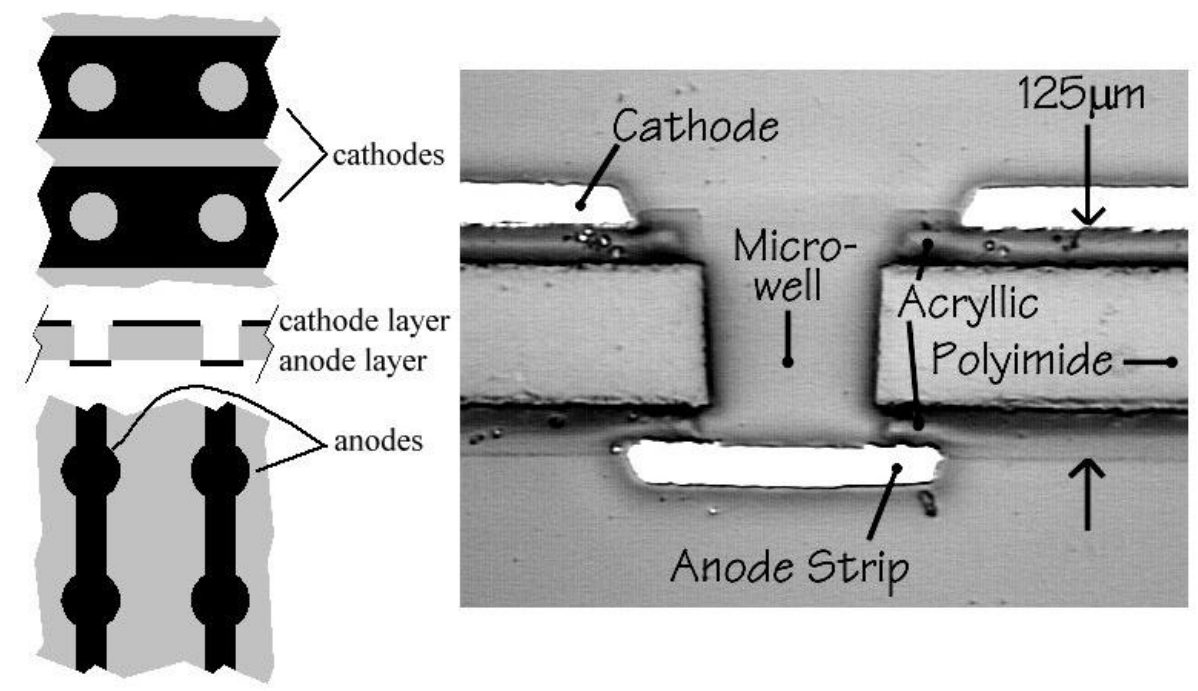

Figure 1. Micro-well detectors. Left: Copper cathode (top) and anode (bottom) traces for crossed-strip design. Wells are formed by exposing the anode through the cathode and substrate. Right: Cross section of an individual well formed by UV laser ablation with a polymide substrate. ${ }^{10,11}$

\section{THREE-DIMENSIONAL TRACK IMAGING DETECTORS}

\subsection{Gas Micro-Well Detectors}

The gas micro-well detector ${ }^{10-12}$ (MWD) is a type of gas proportional counter based on micro-patterned electrodes. Each sensing element consists of a charge-amplifying well, as shown in Figure 1. The cathode and anode electrodes are deposited on opposite sides of an insulating substrate. The well is formed as a cylindrical hole through the cathode and substrate, exposing the anode. An array of such wells forms a detector. The active tracking volume is bounded by a drift electrode on one side and the wells on the other. Ionization electrons produced by the passage of a high-energy charged particle drift toward the anodes and into the wells. An ionization avalanche occurs in each well, where there is an intense electric field set up by the voltage applied between the anode and cathode. The electrons from the avalanche are collected on the anode, while an equal but opposite image charge is measured on the cathode. The gas gain from this avalanche provides the initial charge amplification.

Goddard Space Flight Center has been producing MWDs for the past several years on rugged polymide substrates using UV laser ablation. ${ }^{10,11}$ These detectors have been constructed in a crossed-strip geometry, with the cathodes and anodes forming orthogonal strips on opposite side of the substrate, to allow simple twodimensional imaging at X-ray energies. Very good detector performance has been observed, ${ }^{10-12}$ including stable operation at gas gains of $3 \times 10^{4}$ in Ar- and Xe- based gases, the ability to sustain repeated breakdowns without damage, a FWHM energy resolution of $18 \%$ for $5.9 \mathrm{keV}$ X-rays in $95 \% \mathrm{Xe} / 5 \% \mathrm{CO}_{2}$ gas, and a $\mathrm{FWHM}$ position resolution of $\sim 200 \mu \mathrm{m}$ for $400 \mu \mathrm{m}$ well pitch. For the much larger-area detectors needed for gamma-ray telescopes, different fabrication and readout methods are required (see below).

\subsection{Micro-Well Detectors for a Three-Dimensional Track Imager}

The Three-Dimensional Track Imager (3-DTI) is a concept for a time projection chamber (TPC) with a large area two-dimensional, crossed-strip MWD readout layer (Figure 2). Charged particles traversing the TPC volume leave a track of ionization in the gas. This ionization charge drifts towards the MWD layer and into individual micro-wells where it produces an avalanche and thus the signals on the anode and cathode electrodes. The pattern of the wells which produce the signals is a two-dimensional projected image of the ionization (Figure 3). The third spatial dimension is obtained by timing the drift of the ionization charge. The signal from each anode 


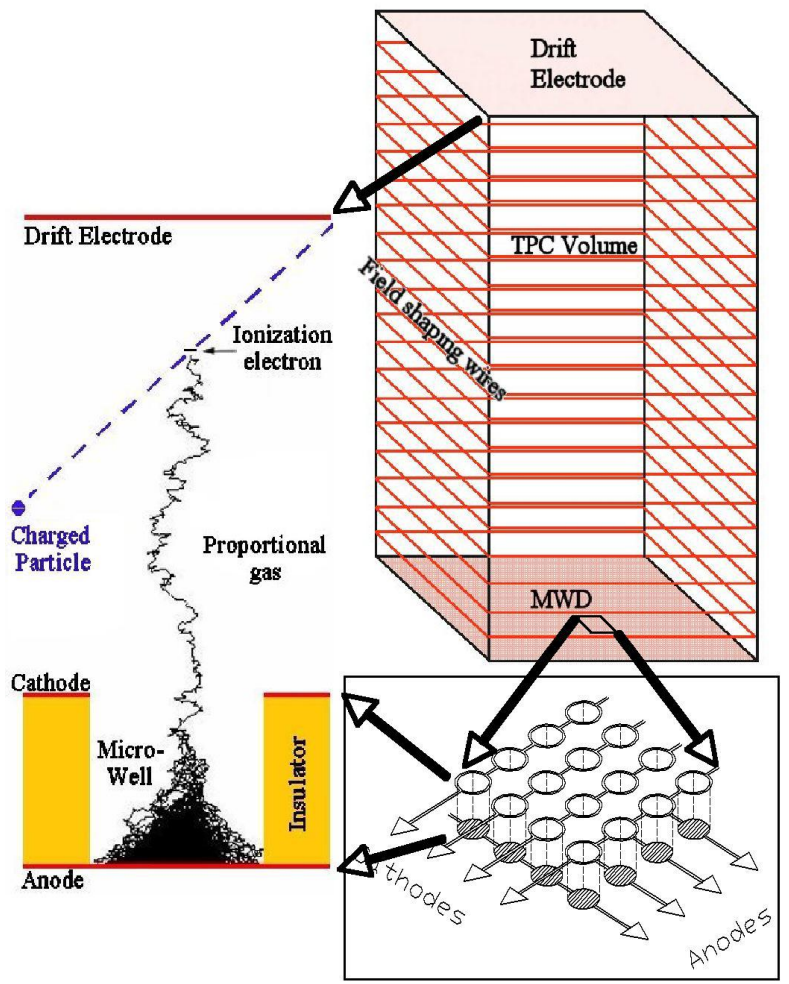

Figure 2. The three-dimensional track imager. Energetic charged particle generate a track of charge which drifts into the wells of the MWD. Recording the time signature of the signals on the $\mathrm{X}$ and $\mathrm{Y}$ strips allows full 3 -D reconstruction of the original track.

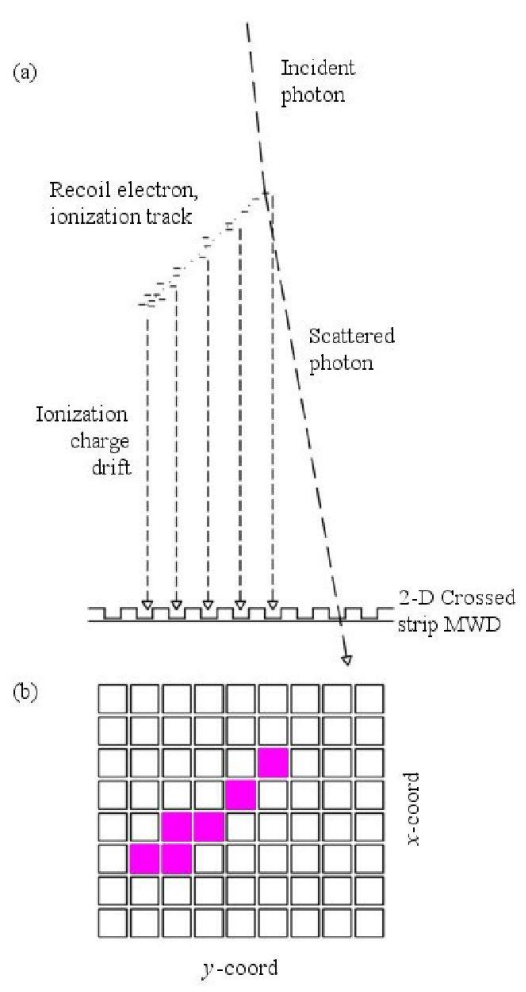

Figure 3. The pattern of wells in which an avalanche occurs in the MWD form a projected image of the original charged particle track. The third dimension is obtained by timing the relative drift of the charges.

and cathode strip is first amplified by the front-end electronics (FEE) and then its time signature is recorded with a transient digitizer (TD). The $\mathrm{X}$ and $\mathrm{Y}$ digitized signals give the $\mathrm{X}-\mathrm{Z}$ and $\mathrm{Y}-\mathrm{Z}$ projected track images from which the three-dimensional track information can be reconstructed (Figure 5).

The 3-DTI detector represents a departure from medium- and high-energy gamma-ray detectors currently under development. For example, the Medium Energy Gamma-ray Astronomy (MEGA) telescope ${ }^{13}$ utilizes double sided silicon strip detectors to provide the Compton scattering medium and to track the recoil electron. A similar approach is used in the GLAST-LAT at high energies: tracking of the electron/positron pair is done with pairs of orthogonal, single-sided silicon strips. Lead foils are interleaved with the silicon layers to provide the pair conversion medium. These approaches have a high density per measurement layer: 3.2 milli-radiation lengths (mRL) per layer for MEGA (0.3 mm Si per layer), and $26 \mathrm{mRL}$ per layer for GLAST (LAT upper tracker, $2 \times 0.3 \mathrm{~mm} \mathrm{Si}+1.12 \mathrm{~mm} \mathrm{~Pb}$ ). This contributes significant scattering to the electrons as they traverse the tracking layers. The active volume of 3-DTI detector, on the other hand, has no passive material and the interaction material is provided by the drift gas. For example, the 3-DTI, filled with xenon at $3 \mathrm{~atm}$, has a density of $8 \times 10^{-5}$ RL per $400 \mu \mathrm{m}$ measurement resolution interval. Thus the 3-DTI provides several tens to hundreds of track measurements of the recoil or pair electrons before their direction is confused by scattering equivalent to even one MEGA or LAT track measurement layer.

\subsection{Realization of the 3-DTI Detector}

The combination of two-dimensional MWDs with transient digitizer readout provides several advantages for the 3-DTI. The active volume is determined by the geometric area of the MWD (i.e. the number of anode + cathode electrodes) and the drift distance. The active volume is limited by the number of FEE channels (i.e. the power 


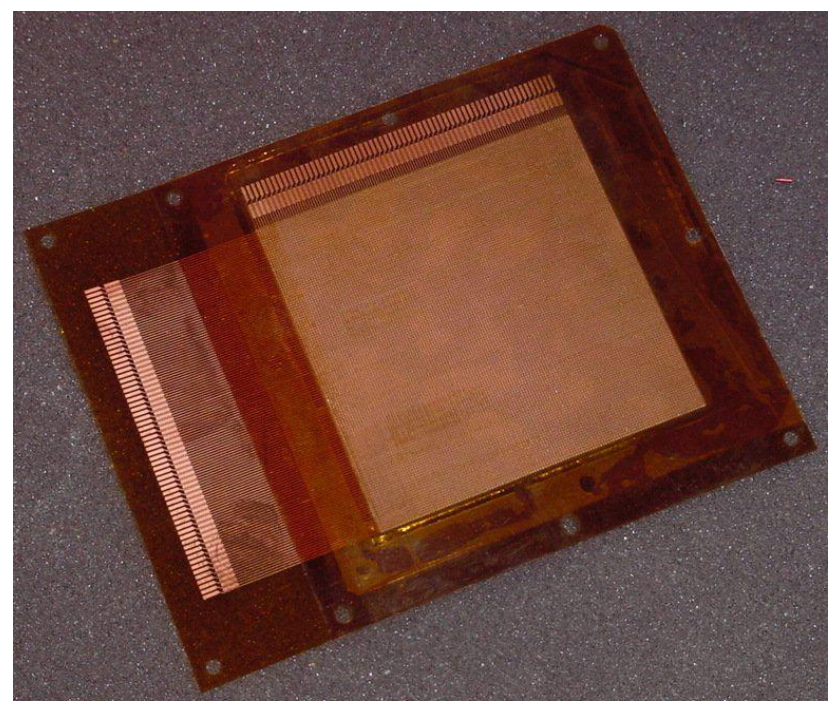

Figure $4.5 \mathrm{~cm} \times 5 \mathrm{~cm}$ MWD produced by Mikro Systems Inc.

available for the FEE and TDs) and the acceptable amount of diffusion of the ionization charge while traversing the gas.

Our current FEE and TD designs, described below, require $\sim 10 \mathrm{~mW}$ and $\sim 35 \mathrm{~mW}$ per channel respectively. Thus, our goal of a $1 \mathrm{~m}^{3} 3$-DTI with $400 \mu \mathrm{m}$ pitch would have $5000 \mathrm{FEE}$ and TD channels and require $\sim 225 \mathrm{~W}$ per MWD layer.

Electrons drifting in $95 \%$ xenon $+5 \% \mathrm{CO}_{2}$ have a minimum transverse diffusion coefficient $\sigma_{x} \sim 0.2 \mathrm{~mm}$ per $\sqrt{\mathrm{cm}}$ of drift in an electric field of $0.25 \mathrm{~V} \mathrm{~cm}^{-1}$ torr $^{-1}$ and a drift velocity of $2 \times 10^{4} \mathrm{~m} \mathrm{~s}^{-1}{ }^{14}$ This non-thermal diffusion limits the maximum drift distance to $\sim 5 \mathrm{~cm}$ before the spatial information of the ionization charge is lost. Our $1 \mathrm{~m}^{3}$ detector would then require $20 \mathrm{MWD}$ layers and a total power of $1125 \mathrm{~W}$.

This total power is unacceptable for a space flight instrument and must be reduced by a factor of $\sim 20$. The FEE and TD designs have not been optimized for minimum power; however, we do not expect reductions of more than a factor of $\sim 2$. An alternative approach is to increase the maximum drift distance by reducing the diffusion coefficient to a value closer to the thermal diffusion limit. Free electrons drift super-thermally; however, negative ion molecules remain in thermal equilibrium with the gas and, hence, have much lower diffusion. Carbon disulfide, $\mathrm{CS}_{2}$, is a moderately electro-negative molecule that has been shown to quickly scavenge the ionization charge, form negative ions that drift thermally, and, in the strong electric field of the micro-well, give up the electrons so that they produce a normal electron avalanche in the well. ${ }^{15,16}$ The admixture of $\mathrm{CS}_{2}$ to argon reduces the drift velocity to $\sim 20 \mathrm{~m} \mathrm{~s}^{-1}$ at $4 \mathrm{~V} \mathrm{~cm}^{-1} \mathrm{torr}^{-1}$. The transverse diffusion coefficient is also greatly reduced. For $75 \% \mathrm{Ar}+25 \% \mathrm{CS}_{2}, \sigma_{x} \sim 0.008 \mathrm{~mm}$ per $\sqrt{\mathrm{cm}}$ of drift. ${ }^{15,16}$ Similar reductions are expected for xenon $+\mathrm{CS}_{2}$ mixtures. The dramatic decrease in $\sigma_{x}$ due to this "negative ion drift" (NID) readily allows for an increase in the maximum drift distance to $>100 \mathrm{~cm}$ for a single MWD layer.

\subsubsection{Detector Fabrication}

The 3-DTI detector requires fabrication of MWDs with $\sim 1 \mathrm{~m}^{2}$ areas. We are working with Mikro Systems Inc. to apply their proprietary micro-casting and laminating technologies to large area MWD fabrication. The laser ablation technique is not viable for detectors larger than a few hundred $\mathrm{cm}^{2}$. We are currently testing $5 \mathrm{~cm} \times 5$ $\mathrm{cm}$ MWDs with a pitch of $400 \mu \mathrm{m}$ in the laboratory, and expect $10 \mathrm{~cm} \times 10 \mathrm{~cm}$ MWDs in a few months and 30 $\mathrm{cm} \times 30 \mathrm{~cm}$ MWDs in about one year. The $5 \mathrm{~cm} \times 5 \mathrm{~cm}$ currently undergoing testing is shown in Figure 4 . 


\subsubsection{Front-end Electronics}

We are currently designing our multi-channel front-end electronics (FEE) using discrete components rather than developing an ASIC. This approach offers several advantages: a flexible, easily modified design, much lower cost, and faster development time. Disadvantages are that each FEE channel is physically much larger and consumes much more power than an ASIC. Once the FEE is tested and optimized we will design a low-power, multi-channel ASIC to match the discrete FEE performance.

\subsubsection{Transient Digitizer}

The drift velocity of negative $\mathrm{CS}_{2}$ ions in xenon is up to $50 \mathrm{~m} \mathrm{~s}^{-1}$, depending on the reduced drift field. A spatial resolution of $400 \mu \mathrm{m}$ in the drift direction, equivalent to the micro-well pitch, requires a Nyquist sample rate of $2 \times\left(50 \times 10^{2} \mathrm{~cm} \mathrm{~s}^{-1}\right) / 0.04 \mathrm{~cm}=250 \mathrm{kHz}$. Over-sampling by a factor of ten, $2.5 \mathrm{MHz}$, is readily achievable with modern ADCs and will allow detailed reconstruction of the charge from multiple particle tracks. Our multichannel TD has 48 12-bit ADC channels and a FPGA that provides the sample clock, trigger detection, cyclic data buffer, and data readout. A trigger condition, on each TD board, is detected by a FPGA as the ADC data is buffered. The trigger condition is tentatively defined as three or more adjacent TD channels with coincident samples above an adjustable threshold. A trigger condition results in an additional number of ADC samples corresponding to the maximum drift time (about $90 \%$ of the cyclic buffer depth) being recorded; the sampling then stops and a trigger signal is sent to a PC. The PC performs an additional, higher level, trigger evaluation based on all of the TD board trigger information. The entire buffer from each TD board contributing to the trigger is read out by the $\mathrm{PC}$ via a digital I/O card, and the sampling is restarted. No attempt has been made at this time to do any data suppression. Significant data reduction should be possible. The entire TD buffer can be read out in $\sim 100 \mathrm{~ms}$.

\subsubsection{Scintillation Trigger}

Relative channel-to-channel timing of the ionization charge arrival at (and avalanche in) the wells provides sufficient information to reconstruct the three-dimension direction vectors of the tracks. However, a prompt interaction trigger is required to determine the absolute vertical coordinate of the interaction and to allow coincident measurements with other detectors (required for Compton telescope applications). The scintillation flash produced in xenon, the principal component of the drift gas for a gamma-ray telescope, by the recoil electron provides the prompt trigger information needed. We are testing VUV avalanche photodiodes ${ }^{17}$ for this purpose.

\subsection{Three-Dimensional Track Imaging Simuations}

We have done simple simulations of the energy deposit, drift, avalanche, and signal generation in the TD electronics to demonstrate the feasibility of three-dimensional track reconstruction in the 3-DTI detector. The results are in Figure 5. Ionization charges were generated in the shape of a pair-production event. Coulomb scattering was not included in the placement of the charges, but over such a small distance $(15 \mathrm{~mm})$ this should be negligible. The effects of energy resolution and NID charge diffusion were applied, and the signals expected on the TD electronics were simulated for each strip. Then a simple contouring of the TD signals was able to recover the $\mathrm{X}-\mathrm{Z}$ and $\mathrm{Y}-\mathrm{Z}$ projections of the original tracks.

\section{TRACK IMAGING DETECTORS IN ADVANCED GAMMA-RAY TELESCOPES}

We have performed Monte Carlo simulations of two advanced gamma-ray telescope concepts to demonstrate the advantages of three-dimensional track imaging capabilities for improved background rejection, angular resolution, and polarization sensitivity. The two applications of gas tracking detectors are: 1) A tracker for an Advanced Compton Telescope (ACT) in which the recoil electron from the initial Compton scatter may be accurately tracked, greatly reducing the telescope's point spread function; and 2) an Advanced Pair Telescope (APT) whose angular resolution is limited primarily by the nuclear recoil and which achieves useful polarization sensitivity near $100 \mathrm{MeV}$. 

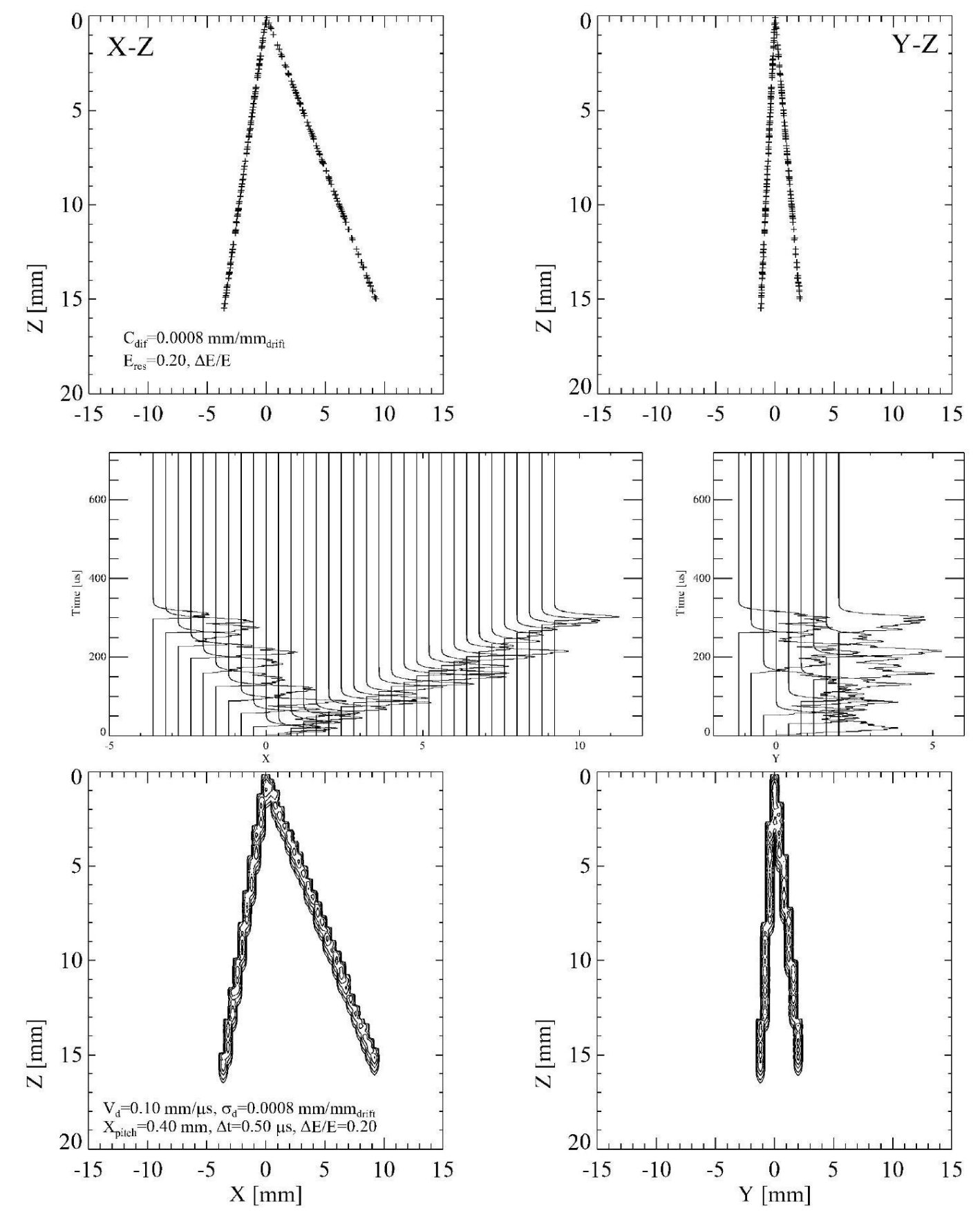

Figure 5. Simple simulations of three-dimensional track reconstruction. Charges were deposited in the shape of a pairproduction event (top). The effects of energy resolution and charge diffusion appropriate for NID were applied, and the signals recorded on the TD electronics were simulated (middle). Simple contouring of the TD signals easily recovers the $\mathrm{X}-\mathrm{Z}$ and Y-Z projections of the tracks (bottom). 


\subsection{Advanced Compton Telescope}

\subsubsection{Science Goals and Advantages of Electron Tracking}

The Advanced Compton Telescope ${ }^{18}$ is envisioned as a medium-energy gamma-ray mission with a $\sim 100$-fold increase in sensitivity over that of COMPTEL, the only Compton telescope that had enough sensitivity to make astronomical observations. ${ }^{1}$ The primary science goal of ACT is the study of gamma-ray lines from Type Ia supernovae (SNe Ia). In particular, the decay lines of ${ }^{56} \mathrm{Co}$ at $812 \mathrm{keV}, 847 \mathrm{keV}$, and $1.238 \mathrm{MeV}$ are important diagnostics of the SN Ia explosion mechanism. The lines are expected to be Doppler-broadened by 3-4\% FWHM. A broad-line sensitivity of a few $\times 10^{-7}$ photons $\mathrm{cm}^{-2} \mathrm{~s}^{-1}$ in $10^{6} \mathrm{~s}$ together with an energy resolution of a few percent or better would enable ACT to detect 50-100 SNe Ia per year, 10 of them with enough significance to distinguish between Chandrasekhar and sub-Chandrasekhar mass models. ${ }^{19}$ Other science goals include the study of radioactive decay lines from supernova remnants, classical novae, and nucleosynthesis in massive stars; the mapping of galactic and extragalactic diffuse gamma-ray emission; the study of the medium-energy behavior of black holes, pulsars, and active galactic nuclei (AGN); and the study of gamma-ray lines from nuclear interactions in solar flares. Compton telescopes are inherently sensitive to polarization, ${ }^{20,21}$ and so a particularly groundbreaking science goal is the study of the gamma-ray polarization properties of gamma-ray bursts, pulsars, solar flares, and AGN.

Part of the required $100 \times$ increase in sensitivity can be achieved by accepting larger Compton scatter angles, increasing the effective area. The rest will have to come from a dramatic decrease in the telescope PSF, which reduces the area of the sky from which a given source's photons could have originated. This will reduce contamination from internal background, from diffuse cosmic and atmospheric gamma-rays, and from nearby astrophysical sources. There are two components to the PSF of a Compton telescope. ${ }^{7}$ The first is the error in the computed scatter angle $\Delta \phi$. (This is often referred to as the angular resolution measure, or ARM.) This width is determined by the spatial and energy resolution of the detectors that make up the telescope. The second component, $\Delta \theta$, is roughly given by the error in the measurement of the recoil electron's initial direction, projected onto the plane perpendicular to the scattered photon direction. COMPTEL was not able to track the recoil electron at all, and so $\Delta \theta=2 \pi$. The PSF for a single photon was thus an annulus on the sky (the "event circle") with the diameter given by the scatter angle $\phi$ and the width by $\Delta \phi$. The total angular area of the PSF, $A=\sin \phi \Delta \phi \Delta \theta$, was therefore quite large for all but the smallest scatter angles. The ACT must accept scatter angles up to $\sim 90^{\circ}$ or greater, and so good electron tracking may well be critical to keep the PSF, and therefore background, within reasonable limits.

\subsubsection{Monte Carlo Simulations and Estimated Performance}

We have studied an electron-tracking ACT concept based on the 3-DTI detector using sophisticated Monte Carlo simulation tools. These tools have been developed as part of NASA's ACT Vision Mission Concept Study with the goal of evaluating and comparing different ACT detector technologies within a common framework. The tools are based on the MGGPOD simulation package ${ }^{22}$ (itself based on the GEANT3 Monte Carlo package ${ }^{23}$ ) and advanced Compton event reconstruction techniques. ${ }^{24}$ The interactions of gamma rays and particles with matter are simulated, including delayed emission from radio-activation, the appropriate detector response is applied, and Compton events are reconstructed from the simulated energy deposits. In this way a given telescope concept's response to astrophysical sources and to photon and particle background sources may be evaluated, and thus its sensitivity estimated. For the purposes of the ACT Concept Study the telescope must have a mass of less than $1850 \mathrm{~kg}$, fit within an envelope of $2 \mathrm{~m} \times 4.4 \mathrm{~m} \times 14 \mathrm{~m}$, and use less than $1730 \mathrm{~W}$ of power. The $847 \mathrm{keV}$ line of ${ }^{56} \mathrm{Co}$ has been selected as a basis for comparison.

Our ACT concept uses a gas 3-DTI tracker to record Compton scatter events and track the recoil electron, and a scintillator-based calorimeter to absorb the scattered photon. The tracker consists of $2 \times 2 \times 43$-DTI modules, each $80 \mathrm{~cm} \times 80 \mathrm{~cm}$ MWD area $\times 50 \mathrm{~cm}$ drift length. The walls of the modules are made of polyamide and $\mathrm{Cu}$ held by a carbon fiber composite support frame. The tracker is full of $97 \% \mathrm{Xe}+3 \% \mathrm{CS}_{2}$ gas at $3 \mathrm{~atm}$, and we assume a drift diffusion of $\sigma_{x}=0.001 \mathrm{~mm} \times \sqrt{\operatorname{drift}(\mathrm{cm})}$ appropriate for NID. ${ }^{15,16}$ We assume a pitch of $200 \mu \mathrm{m}$ and the energy resolution given in Section 2.1 scaled by the square root of the energy. The calorimeter is made of $\mathrm{LaBr}_{3}$, a new scintillator material with high density, fast timing, and excellent energy resolution. ${ }^{25}$ We assume $5 \mathrm{~mm} \times 5 \mathrm{cmm}$ crystals, $4 \mathrm{~cm}$ thick below the tracker and $2 \mathrm{~cm}$ thick on walls which extend 90 


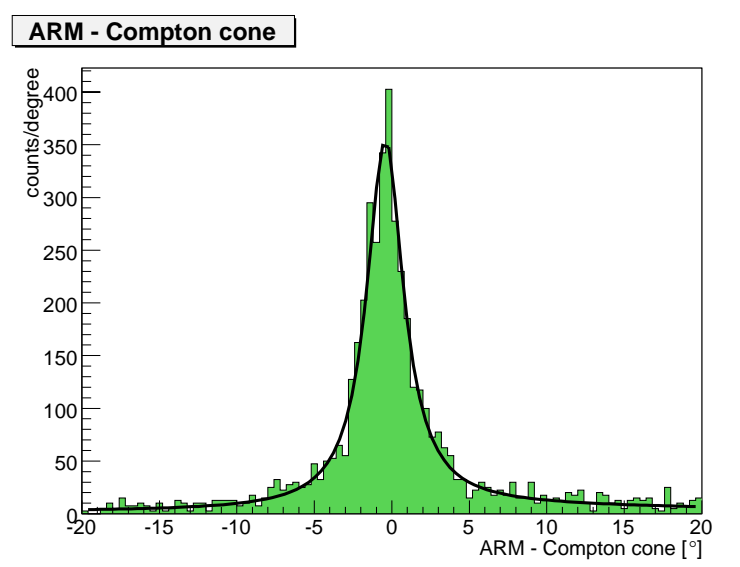

Figure 6. ACT ARM distribution for events within a $836-870 \mathrm{keV}$ window. $\mathrm{FWHM}=2.8^{\circ}$.

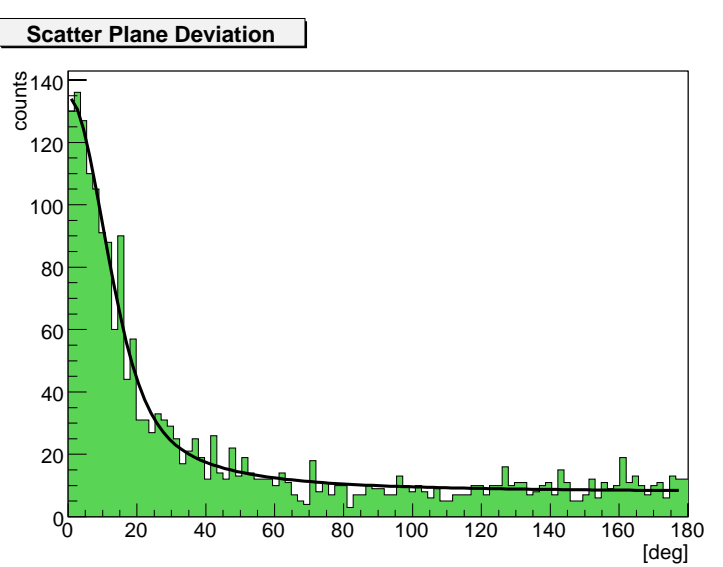

Figure 7. ACT SPD distribution for all events within a $836-870 \mathrm{keV}$ window. The $2.35 \sigma$ width is $27.1^{\circ}$.

$\mathrm{cm}$ up the sides. We assume an energy resolution of $3 \% \mathrm{FWHM}$ at $662 \mathrm{keV} .{ }^{25}$ A coincident trigger between the tracker and calorimeter is achieved via the VUV scintillation light from the xenon gas and the inherent fast signal of the $\mathrm{LaBr}_{3}$.

For our initial ACT evaluation we simulated incident photons with an energy of $847 \mathrm{keV}$. We require at least one hit in both the tracker and calorimeter. We use a very simple track-finding algorithm: the simulated energy deposits are first randomly displaced to simulated the charge diffusion, and then averaged into clusters with a radius of $2 \times$ the pitch. The clusters are then simply strung together by continuously finding the nearest neighbor withing an arbitrary search radius. The start of the track is determined by the expectation that the scattering and average energy deposit will increase along the track as the electron loses energy. ${ }^{24}$ From the recorded energy spectrum of the $847 \mathrm{keV}$ line we find an energy resolution of 3.4\% FWHM. We evaluated the telescope tracking and imaging performance within an energy window of $836-870 \mathrm{keV}$. The angular resolution, defined as the angular resolution measure (ARM), the difference between the calculated and true Compton scatter angle, is shown in Figure 6. The FWHM of the distribution is $2.8^{\circ}$. Another figure of merit for an electron-tracking telescope is the electron scatter plane deviation (SPD), the angle between the measured recoil electron direction and the true plane in which the photon scatters. This is shown for all events within the same total energy window in Figure 7. The width of the distribution, defined here as a "FWHM" or $2.35 \sigma$, is $27.1^{\circ}$. We can also fit the $\mathrm{SPD}$ as a function of electron energy; for a power law fit, we find $\mathrm{FWHM}_{S P D}(\mathrm{deg})=6.1+1845 \times(\mathrm{E}(\mathrm{keV}))^{-0.9}$; this gives an spread of about $10^{\circ}$ for a $1 \mathrm{MeV}$ electron.

We next simulated the response of this ACT concept to a $847 \mathrm{keV}$ line broadened by $3 \%$ for various zenith angles (with $0^{\circ}$ defined as on-axis). We used an energy window of 829-887 keV. We found the effective area of the telescope for two cases: 1) using all events within the energy window and \pm FWHM of the standard gamma-ray ARM distribution, and 2) using only those events within the energy window and $\pm \mathrm{FWHM}$ of the "dual ARM" defined by both the gamma-ray ARM and the electron SPD. The results are shown in Figure 8. The effective area falls off quite slowly with zenith angle, indicating that the telescope has a very wide field of view. The effective area is smaller for the dual ARM at all angles, but this is deceptive since it ignores the background. We have begun background simulations using MGGPOD to determine the total sensitivity. So far we have simulated only the background due to gamma-ray photons from the diffuse cosmic background and from the Earth's atmosphere. Based on the number of photons from these sources that pass the same cuts used to derive the effective areas, we find that the on-axis $3 \sigma$ sensitivity at $847 \mathrm{keV}$ for a $10^{6}$ s observation is $6.2 \times 10^{-6}$ photons $\mathrm{cm}^{-2} \mathrm{~s}^{-1}$ for the standard gamma-ray ARM and $3.7 \times 10^{-6}$ photons $\mathrm{cm}^{-2} \mathrm{~s}^{-1}$ for the dual ARM. Thus the ablility to track electrons provides a $\sim 67 \%$ improvement in sensitivity. We note that a gas-based ACT is the only concept able to track electrons at this low an energy. The derived sensitivity is $\sim 10 \times$ higher than that desired for ACT, but this is largely due to the size and mass constraints imposed for the Concept Study simulations. A larger tracker, surrounded by a thicker calorimeter, would greatly increase the effective area of 


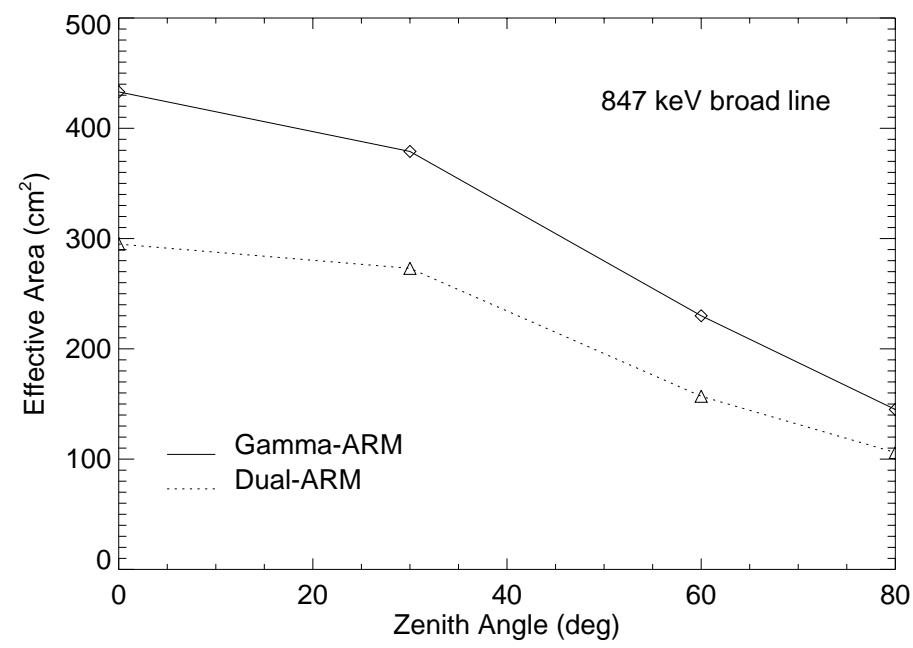

Figure 8. Effective area of the ACT concept for a broad $847 \mathrm{keV}$ line as a function of incident angle. The area is plotted for both the "standard" gamma-ray ARM and the "dual ARM," which includes the electron SPD.

the telescope, with an obvious corresponding increase in the requirements for the launch vehicle.

\subsection{Advanced Pair Telescope}

\subsubsection{Science Goals and High-Energy Polarimetry}

Numerous science goals for high-energy $(30 \mathrm{MeV}->50 \mathrm{GeV})$ gamma-ray astrophysics require greatly improved angular resolution over past or currently planned missions such as GLAST. The most basic of these goals is a complete census of high energy sources in the Galaxy, including a definitive distinction between classes of point sources and truly diffuse emission. Most of the EGRET point sources are still unidentified; accurately locating even faint sources will vastly simplify the identification of counterparts and allow statistically significant studies of source populations. High resolution mapping of diffuse emission will allow detailed comparison with radio maps of molecular and atomic hydrogen gas, which in turn will reveal the distribution of cosmic rays in the interstellar medium. ${ }^{26}$ Nearby supernova remnants will be mapped, permitting the study of electronic vs. hadronic emission models. For the first time external galaxies (e.g. M31) will be mapped in gamma-rays, allowing their source populations and cosmic ray distributions to be determined. ${ }^{27}$ Within the solar system, the high-energy components of solar flares will be localized, constraining models of particle acceleration. ${ }^{28}$ These science goals will require an angular resolution roughly an order of magnitude better than that of GLAST, from $<0.5^{\circ}$ below $100 \mathrm{MeV}$ to a few arcminutes near $1 \mathrm{GeV}$. Such resolution for single photons is possible using telescopes based on gas detectors. Above $30 \mathrm{MeV}$, gamma-ray telescopes form images by reconstructing the tracks of the electron and positron formed by pair production. The angular resolution of a pair production telescope is limited by the multiple scattering of the electron and positron in the detector material and by the unknown recoil of the particle (nucleus or electron) in whose field the pair conversion took place. It has been shown that a pair telescope can nearly achieve recoil-limited resolution, approaching 1 arcmin above a few GeV, if the density of the tracking medium can be made less than $\sim 2 \times 10^{-5} \mathrm{RL}$ per track measurement interval. ${ }^{6}$ In addition, a fraction of the pair conversions will take place in the field of an electron, ${ }^{29}$ and the track of this recoil electron will also be measurable in a low-density detector medium, allowing complete kinematic reconstruction of the event. The cross section for this so-called triplet production process is reduced by a factor of $1 / Z$ compared to nuclear pair production.

Polarization sensitivity will provide a new tool for high-energy gamma-ray astrophysics. Polarimetry provides information on source geometry, particularly anisotropies due to magnetic fields and particle distributions. Polarization measurements will thus permit study of emission mechanisms in gamma-ray pulsars, bright active 


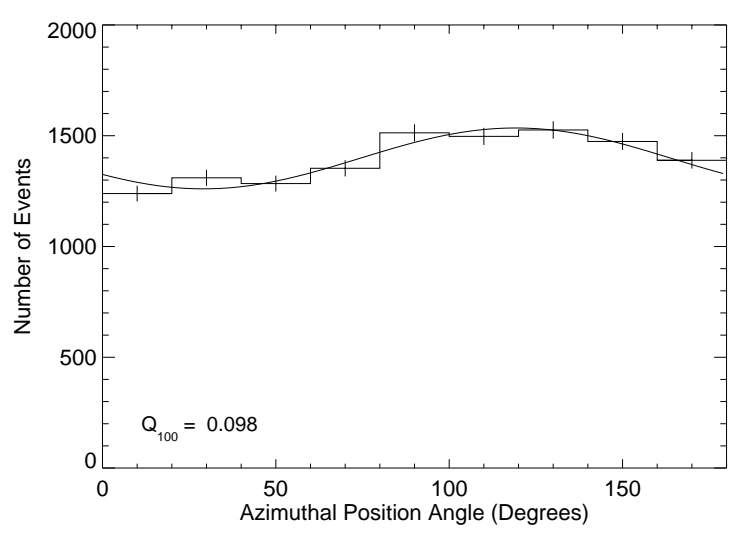

Figure 9. True APT azimuthal distribution for $100 \%$ polarized, $100 \mathrm{MeV}$ photons, taken directly from the GEANT4 simulation output before the detector response is applied. Measued polarization angle is shifted by $\sim 9^{\circ}$ due to the effects of non-coplanar events. Modulation factor is 0.098 .

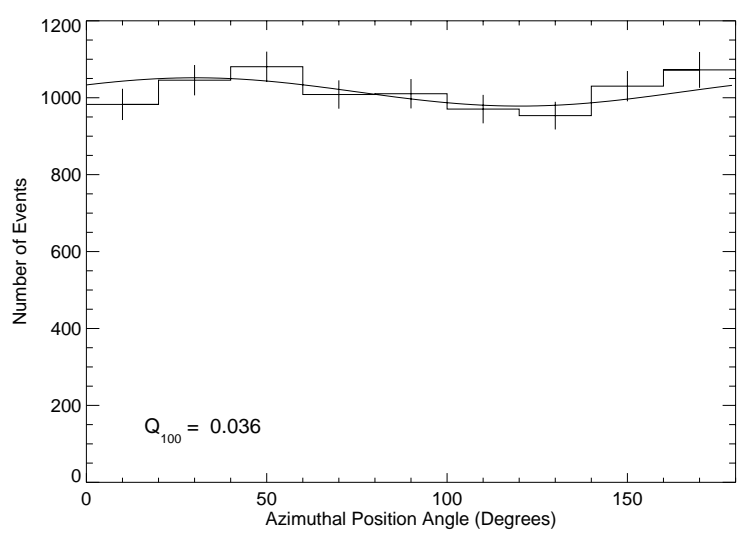

Figure 10. Reconstructed APT azimuthal distribution, including the effects of the detector response and track fitting. The fitted modulation factor of 0.036 is not significant.

galactic nuclei, high-energy gamma-ray bursts, and allow the distinction of electron bremsstrahlung emission from pion decay in solar flares. Polarimetry is in principle possible with pair production telescopes due to the fact that the azimuthal orientation of the electron-positron plane is weakly correlated with the incident photon's electric field vector. ${ }^{30}$ Past and currently-planned pair telescopes such as EGRET and GLAST, however, have negligible polarization sensitivity due to the multiple scattering of the pair particles in the thick converter foils, which quickly masks the original plane of the pair. ${ }^{5,31}$ We have previously shown that a pair telescope based on gas detectors should in principle be sensitive to polarization from bright sources at $\sim 100 \mathrm{MeV} .{ }^{8}$

\subsubsection{GEANT4 Simulations and Estimated Performance}

We have performed Monte Carlo simulations of an Advanced Pair Telescope concept based on the 3-DTI detector. Because pair production of polarized gamma rays is not implemented in GEANT3, we have used GEANT4 ${ }^{32}$ for these simulations. Polarized pair production has been implemented in GEANT4 by G. Depaola and F. Longo, ${ }^{33-35}$ and we have previously used their pair production class to evaluate a preliminary APT design. ${ }^{8}$ Although these simulations were performed with GEANT4 v5.1, we have used the multiple scattering class from v3.2, provided by T. Burnett of the GLAST collaboration, due to known bugs in the more recent versions.

The APT concept was simulated using $1 \mathrm{~m}^{3}$ 3-DTI modules divided down the middle by a drift electode, giving two $100 \mathrm{~cm} \times 100 \mathrm{~cm}$ MWD area $\times 50 \mathrm{~cm}$ drift volumes. The modules were filled with $94 \%$ Ar $+6 \%$ $\mathrm{CS}_{2}$ gas at $3 \mathrm{~atm}$; Ar was used instead of Xe to maximize the cross section for triplet production, although this is not yet implemented in GEANT4. Eight modules were placed in a stack for a total length of $8 \mathrm{~m}$, or $\sim 0.25$ RL. A complete telescope would be formed by grouping many such stacks side-by-side. A massive calorimeter, as in GLAST, is not required, since the energy of the pair particles may be estimated by their average degree of scattering. ${ }^{6}$ Between each 3-DTI module was inserted $3 \mathrm{~mm}$ of plastic scintillator to act as a triggering detector, with another plastic layer over the aperture to act as an anticoincidence shield. The basic trigger condition is $>400 \mathrm{keV}$ energy deposit in at least two consecutive scintillators in anticoincidence with the outer shield. 100 $\mathrm{MeV}$ photons, $100 \%$ polarized, were simulated entering the APT stack on-axis with a polarization angle of $110^{\circ}$. For each event the same detector response (energy resolution and charge diffusion) was applied as for the ACT concept (Section 3.1.2). Then the two longest and straighest tracks were found and fitted with straight lines near the vertex. The photon incident direction was found by the energy-weighted addition of these two fitted vectors, and the azimuth angle of the plane formed by the two vectors was calculated.

The results of the simulations are shown in Figure 9 and Figure 10. Figure 9 shows the histogram of pair plane azimuth angles obtained directly from the raw simultion before applying the detector response and event 
reconstruction. This is, in effect, the "best possible case" result. A clear azimuthal modulation due to the polarized input is evident, and the modulation factor, defined as the (maximum - minimum)/(maximum + minimum) of a sinusoidal fit, ${ }^{8}$ is $Q_{100}=0.098$. The measured polarization angle is $119.1^{\circ}$; the shift from the input value is due to the effect of non-coplanar pair events and should be correctable. ${ }^{8}$ The angular resolution, defined as the angular radius containing $68 \%$ of all events, is $\Theta_{68}=0.6^{\circ}$. Figure 10 shows the same azimuthal histogram after the detector response and event reconstruction has been applied. Although the histogram still appears modulated, the angle is incorrect and the fitted modulation, $Q_{100}=0.036$, is not significant. The angular resolution is $\Theta_{68}=1.3^{\circ}$. We believe this degradation in the telescope performance is due to problems with our simple track fitting procedure near the vertex, where the information about the initial particle momenta is preserved. We are currently developing a new track reconstruction fitting procedure based on maximum-likelihood fitting.

\section{CONCLUSIONS AND FUTURE WORK}

We have demonstrated that three-dimensional track imaging detectors based on gas MWD hold great promise for future medium- and high-energy gamma-ray detectors. The development of MWDs into 3-DTI detectors is currently supported under a NASA APRA program at NASA/GSFC. This program supports the development of MWDs and their readout electronics as well as investigations of optimum gas mixtures, event reconstruction algorithms, and Monte Carlo simulations. A small prototype will be tested at a polarized gamma-ray beam within three years. Further technology development will be needed to scale up MWD production and readout electronics to cover large areas, and to test larger prototypes at accelerator beams and on scientific balloon flights.

\section{ACKNOWLEDGMENTS}

This work is partially supported by the NASA APRA program. We thanks G. Depaola and F. Longo for the GEANT4 pair polarization class and T. Burnett for the modified GEANT4.3.2 multiple scattering class.

\section{REFERENCES}

1. V. Schönfelder, et al., "Instrument description and performance of the imaging gamma-ray telescope COMPTEL aboard the Compton Gamma-Ray Observatory," Astrophysical Journal Supplement Series 86, pp. 657$692,1993$.

2. G. Kanbach, et al., "The project EGRET (energetic gamma-ray experiment telescope) on NASA's gammaray observatory (GRO)," Space Science Reviews 49, pp. 69-84, 1988.

3. M. Tavani, et al., "The AGILE instrument," in X-Ray and Gamma-Ray Telescopes and Instruments for Astronomy, J. E. Truemper and H. D. Tananbaum, eds., Proc. SPIE 4851, pp. 1151-1162, 2003.

4. P. F. Michelson, "Instrumentation for the gamma-ray large area space telescope (GLAST) mission," in $X$ Ray and Gamma-Ray Telescopes and Instruments for Astronomy, J. E. Truemper and H. D. Tananbaum, eds., Proc. SPIE 4851, pp. 1144-1150, 2003.

5. J. R. Mattox, "The sensitivity of EGRET to gamma-ray polarization," Exp. Astron. 2, pp. 75-84, 1991.

6. S. D. Hunter, D. L. Bertsch, and P. Deines-Jones, "Design of a next generation high-energy gamma-ray telescope," in Gamma 2001, S. Ritz, N. Gehrels, and C. R. Shrader, eds., AIP Conf. Proc. 587, pp. 848$852,2001$.

7. P. F. Bloser, et al., "Applications of gas imaging micro-well detectors to an advanced Compton telescope," New Astronomy Reviews 48, pp. 299-303, 2004.

8. P. F. Bloser, S. D. Hunter, G. O. Depaola, and F. Longo, "A concept for a high-energy gamma-ray polarimeter," in X-Ray and Gamma-Ray Instrumentation for Astronomy XIII, K. A. Flanagan and O. H. W. Siegmund, eds., Proc. SPIE 5165, pp. 322-333, 2004.

9. P. F. Bloser and S. D. Hunter, "Pixelized gas micro-well detectors for advanced gamma-ray telescopes," in Proceedings of the Fifth INTEGRAL Science Workshop, V. Schonfelder, G. Lichti, and C. Winkler, eds., ESA Special Publication SP-552, p. 765, 2005. 
10. J. K. Black, P. Deines-Jones, S. D. Hunter, and K. Jahoda, "Imaging micro-well detectors for X-ray and gamma-ray applications," in X-ray and Gamma-ray Instrumentation for Astronomy XI, K. A. Flanagan and O. H. W. Siegmund, eds., Proc. SPIE 4140, pp. 313-323, 2000.

11. P. Deines-Jones, J. K. Black, H. Crawford, and S. D. Hunter, "Imaging micro-well proportional counters fabricated with masked UV laser ablation," Nucl. Inst. Meth. A 477, pp. 55-58, 2002.

12. P. Deines-Jones, J. K. Black, S. D. Hunter, K. Jahoda, and S. M. Owens, "Large-area imaging micro-well detectors for high-energy astrophysics," Nucl. Inst. Meth. A 478, pp. 130-134, 2002.

13. G. Kanbach, et al., "Development and calibration of the tracking Compton/pair tele scope MEGA," Nuclear Instruments and Methods in Physics Research A 541, pp. 310-322, 2005.

14. A. Peisert and F. Sauli, "Drift and diffusion of electrons in gases: a compilation," Tech. Rep. 84-08, CERN, 1984.

15. C. J. Martoff, et al., "Suppressing drift chamber diffusion without magnetic fields," Nuclear Instruments and Methods in Physics Research A 440, pp. 355-359, 2000.

16. T. Ohnuki, D. P. Snowden-Ifft, and C. J. Martoff, "Measurement of carbon disulfide anion diffusion in a TPC," Nuclear Instruments and Methods in Physics Research A 463, pp. 142-148, 2001.

17. J. A. M. Lopes, et al., "A xenon gas proportional scintillation counter with a UV-sensitive large-area avalanche photodiode," IEEE Transactions on Nuclear Science 48, pp. 312-319, 2001.

18. J. D. Jurfess and R. A. Kroeger, "Progress towards an advanced Compton telescope," in Gamma 2001, S. Ritz, N. Gehrels, and C. R. Shrader, eds., AIP Conf. Proc. 587, pp. 867-876, 2001.

19. P. A. Milne, et al., "Advanced Compton telescope designs and SN science," New Astronomy Reviews 46, pp. 617-623, 2002.

20. F. Lei, A. J. Dean, and G. L. Hills, "Compton polarimetry in gamma-ray astronomy," Space Sci. Rev. 82, pp. 309-388, 1997.

21. M. L. McConnell and J. M. Ryan, "Status and prospects for polarimetry in high energy astrophysics," New Astronomy Reviews 48, pp. 215-219, 2004.

22. G. Weidenspointner, et al., "MGGPOD: a Monte Carlo suite for modeling instrumental line and continuum backgrounds in gamma-ray astronomy," Astrophysical Journal Supplement Series 156, pp. 69-91, 2005.

23. R. Brun, et al., "GEANT detector description and simulation tool," tech. rep., CERN Program Library Long Writeup W5013, 1995.

24. A. Zoglauer, R. Andritschke, and G. Kanbach, "Data analysis for the MEGA prototype," New Astronomy Reviews 48, pp. 231-235, 2004.

25. K. S. Shah, et al., " $\mathrm{LaBr}_{3}$ :Ce scintillators for gamma ray spectroscopy," IEEE Transactions on Nuclear Science 50, p. 2410, 2003.

26. S. D. Hunter, et al., "EGRET observations of the diffuse gamma-ray emission from the galactic plane," Astrophysical Journal 481, p. 205, 1997.

27. P. Sreekumar, et al., "A study of M31, M87, NGC 253, and M82 in high-energy gamma rays," Astrophysical Journal 426, p. 105, 1994.

28. E. L. Chupp, et al., "Future high energy solar missions (imaging $>20 \mathrm{MeV}$ neutral flare emissions)," Advances in Space Research 21, pp. 333-342, 1998.

29. V. F. Boldyshev and Y. P. Peresun'ko, "Electron-positron pair photoproduction on electrons and analysis of proton beam polarization," Sov. Journ. Nuc. Phys. 14, pp. 576-578, 1972.

30. G. C. Wick, "Detection of gamma-ray polarization by pair production," Phys. Rev. 81, pp. 467-468, 1951.

31. J. R. Mattox, H. A. Mayer-Hasselwander, and A. W. Strong, "Analysis of COS B data for evidence of linear polarization of Vela pulsar gamma rays," Astrophys. Jour. 363, pp. 270-273, 1990.

32. S. Agostinelli, et al., "Geant4 - a simulation toolkit," Nucl. Inst. Meth. A 506, pp. 250-303, 2003.

33. G. O. Depaola and C. N. Kozameh, "Detecting polarized gamma rays by pair production," Rad. Phys. Chem. 53, pp. 455-459, 1998.

34. G. O. Depaola, C. N. Kozameh, and M. H. Tiglio, "A method to determine the polarization of high energy gamma rays," Astropart. Phys. 10, pp. 175-183, 1999.

35. G. O. Depaola, "Azimuthal distribution for pair production by high-energy $\gamma$-rays," Nucl. Inst. Meth. A 452, pp. 298-305, 2000. 